\title{
Creating synergy through the Integration of advanced planning systems and configuration
}

\author{
Carsten Svensson ${ }^{1}$ and Kenn Steger-Jensen ${ }^{2}$ \\ ${ }^{1}$ Department of Manufacturing Engineering and Management, Technical University of \\ Denmark, Building 358, DK2800 Kgs. Lyngby, Danmark. csv@ipl.dtu.dk \\ ${ }^{2}$ Department of Production, Aalborg University, Fibigerstraede 16, DK 9220 Denmark. \\ kenn@iprod.auc.dk
}

\begin{abstract}
In recognition of the individuality of customers, manufacturers have through generations presented solutions, which targeted a specific group of customers. In some cases the preferences of the customers are so diversified that it is impossible to include a satisfying number of solutions within a fixed product program. Instead, manufacturers have applied a "customisation" approach. A customisation approach means that the product is partially designed for the customer, and thereby it is possible to adapt the product to fit a very wide range of customers.

Customisation is a challenge to the production system as the opportunity to ensure an efficient utilisation of the production system is reduced, because the "build to order" approach is often a part of customisation. Industrial customisation is significantly different from both mass production and craft production. Therefore the techniques of traditional industrial production can only be applied to a limited extent. This paper will suggest some improvements in information management that could increase transparency in the build to order production that most often characterise customisation.
\end{abstract}

\section{Keywords}

Mass Customization, Advanced Planning and Scheduling (APS), Product Configuration, Production and inventory management.

\section{INTRODUCTION}

Customisation has proven to be a challenge for manufacturers worldwide. Customers are demanding products that are cut to fit, but manufacturers have difficulties manufacturing profitable solutions. All too often manufacturers are struggling with cost, delivery and quality of the customised products, the reasons for this can be numerous, but some of them are related to the operations management. Especially the planning task has become too complicated to be carried out on a daily basis, due to the large number of variables. In order to compensate for this large buffers have been introduced, and profits have been hollowed out by alterations, rush orders, lack of components etc. The daily focus is on getting products out in time rather than on efficiency. As competition intensifies the differentiation value of 
customisation decreases, and in some markets customisation will gradually become a qualifying attribute rather than creating a competitive advantage.

The justification for the existence of the build to order manufactures used to be the ability to deliver customised solutions that offer an increased value to the customers through innovative design and responsiveness to the preferences of the individual customer. Already today manufacturers find that they are being squeezed by traditional mass producers who are adding customisation to their high-end products. The traditional mass producers are not targeting the market for pure customised products, but are focusing on the market for products adapted to the customer. This is done through a customised standardisation [Lampel \& Mintzberg 1996], as illustrated in Figure 1. The traditional customizers are also facing a challenge from globalisation as described by [Lewitt 1983]. The internal rivalry is increasing as hungry manufacturers are looking for new feeding grounds, and as a result customizers will have to revise their perception of efficiency, based on a global rather than a local understanding.

The solution presented in this paper will enable manufacturers of customized products to use production data in the sales situation. By using production data in the sales situation it will be possible to improve the utilization of an already existing production system, thereby reducing cost and lead-time. The approach is based on the observation from a number of medium sized manufacturers. These manufacturers have all established a differentiation by offering a customization of complex products. But this differentiation is not with out costs. As a result of the increased product complexity and customization the efficiency is low.

\section{EFFICIENCY ASPECT OF MASS CUSTOMIZATION}

The manufacturers described in this paper are all medium sized (200 - 500 employees) and produce highly customised products for the world market. From the beginning they have all applied a pure customisation strategy based on the tailored customization production form, as shown in Figure 1.

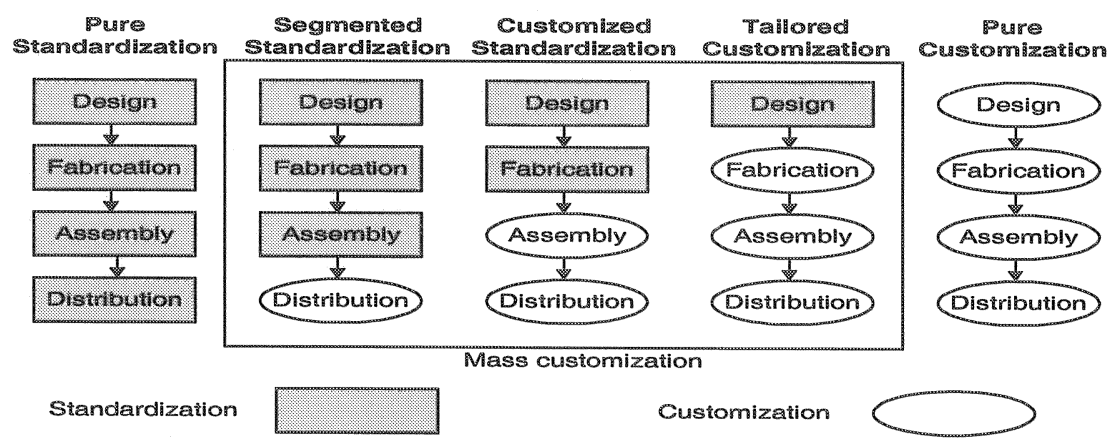

Figure 1 - A continuum of strategies, [Lampel \& Mintzberg 1996]

The uniformity of customers is varying and therefore different strategies are used to meet the need for adaptation. Today, mass producers reach a degree of 
customer satisfaction through a "customised standardisation" strategy equivalent to the one customisers reach through pure customisation.

This craft orientated production approach is currently causing sizeable internal friction, which reduces the competitiveness in relation to larger and more industrialised manufacturers, which are staring to penetrate into the lucrative market for customised products. As a result, the traditional customisers are challenged on their core marked. To meet this challenge, an improved efficiency is needed which can be obtained through reduction in the internal friction. The friction results in the following symptoms:

a Order-processing is time consuming and costly

- Revisions of specifications are multiple

- Through put times are long

- Delivery date are not met

- Last minute changes take up an increasing portion of resources

- Production plans are inaccurate and are often overruled

- As the overturn grows, profits decrease

Typically all of these symptoms are caused by a lack of valid information. Valid information is critical for efficient production of customised goods, but it is also a difficult for manufacturers to provide. Each product is a new combination of complex information regarding a dynamic product, which has become so sophisticated that the data due to the amount cannot be processed and validated manually. This was the case for the highly innovative manufacturer of windturbines, NEG Micon. Despite the fact that NEG Micon is among the five largest manufacturers of wind-turbines, the company has had difficulties with the validity of the production data. Investigations indicated that $55 \%$ of the specifications lacked proper quality [Hvam et al., 2000]. The main focus had been on introduction of new products to the market at a breathtaking speed with little concern about data, and the result was easy see from the bottom-line. In future, focus must be on an industrialisation, which includes a higher degree of structure and standardisation, because most of the variations seen today are non value-adding.

[Pine 1993] suggests that information technology is the key to an efficient production of customised goods, also called mass customisation. Often the information systems of medium sized manufacturers are more suitable for the original craft company rather than and industrial manufacturer, as manufacturers out grow their information systems they become a limiting factor to growth. The manufacturer cannot exceed the limit where a few persons can hold all information regarding a production, and when the limit is reached the mentioned symptoms can be observed.

[Hart 1995] uses the term amenability to describe the enablers for mass customisation. I.e. efficient integration of information processing is one of them, because only through integration will it be possible to manage the information needed for enabling efficient production, especially when it comes to manufacturers who produce a limited number of capital-intensive products. 


\section{PLANNING PROBLEM}

The manufacturers observed produce a limited number of units $(<200$ p.a.). Hence, the individual order is significant in the overall production, and as a result the workload is unstable. In reaction to the unpredictability in both numbers and configurations, manufacturers often turn to a pull-system based on a postponement strategy. The postponement strategy will to some extend reduce lead times and improve the utilisation of the production system, but in general the observed manufacturers are conservative when determining/fixing the push-pull line. The line is fixed according to the longest acceptable delivery time for the customer, and not based on an optimisation of the production in relation to work in progress, numbers etc. As a result, the potential of industrial planning is not fully exploited as the systems are to a large extent based on a pull-system originating from craft production, rather than the traditional industrial push system.

In general, only manufacturers with very high volumes have been able to customise products with the same efficiency as mass producers. E.g. Dell computers and car manufacturers have been successful in applying a postponement strategy. The success is partly due to the very large number of units produced which enables the companies to push products to a high degree of completion before adding the customised features.

\section{CONFIGURATION AND ADVANCED PLANNING SYSTEMS IN RELATION TO CUSTOMISATION}

Both configuration and Advanced Planning Systems (APS-systems) can create great value individually in discrete manufacturing. Integration of APS and configuration will unveil an even larger potential. In a dynamic market for "built to order" production planning is difficult, and this is one of the reasons why "built to order" products in general are more expensive than mass produced products. This extra cost often comes from the sales situation, where the production load is not taken into account because the opportunity lacks rather than the willingness. Accessibility to data is a common problem [Davenport 1999], since sales people are unable to find out what inventory is available for sale as the information only exists in an inaccessible manufacturing system. Therefore the production is largely ignored in the sales process. The problem accrues due to the low vertical integration of IT systems, which means, that it is difficult to link the sales process to the actual situation in the production facility. Sale orders are therefore, to a large extent, processed without consideration of consequences in the production system. The delivery dates presented to customers by the sales department are often based on a static picture of capacity, and prices are based on component prices rather than the actual cost of production. The prices available from configurators are often based on best-case scenarios, which often is not valid in "built to order production". As a result, manufacturers experience that profits are reduced/decreased when production is not running at an optimum. Without the use of both a APS system and configuration system it is a very difficult task to estimate delivery times and include the actual production costs when tenders are given, because the sufficient support for 
a rational decision is not present. These calculations are made every day, and the result is that the tenders are not competitive or not profit generating, since other orders have to be jeopardised to meet the need of a single customer. The limited decision support makes it very difficult to prioritise orders, which is an important function if the manufacturer only produces a relatively small number of products. If the necessary information were available, it would be possible to reschedule more dynamically since the consequences would be evident. The solution presented below will provide information regarding the actual production cost to the configurator, and based on this information it will be possible to calculate the production cost and thereby ensure an acceptable profitability.

\section{CONFIGURATION}

As products move from the uniformity of mass production towards customisation, a need to control the diversity emerges. Every product will become a unique combination of information, which increases the need for information management significantly, since the pure number of rules and data expands dramatically, hence a customisation production needs a huge amount of valid information. To control the diversity in complex products, configurators are often applied to help constrain the cost of the specification process. The specification process covers e.g. the following processes: bids, order management, product configuration [Hvam \& Have 1998].

A configurator is a software tool, which can contain knowledge related to the product. When working with complex customised products, the engineering and validation tasks are often major cost drivers and the efficiency of these processes are critical for the overall competitiveness of the solution provided by the manufacturer. To ensure the efficiency of these processes a formalisation of the rules for the combination of modules or calculations of alterations can advance automation by configurators. Configurators originate from the expert systems but are currently reaching new commercial markets, the breakthrough technology is the constraint based tools which have simplified the maintenance task that constrains the expert systems [Sabin \& Weigel 1998].

One well known example is the simple configurators found at the web where Dell lets us assemble a computer or Mercedes who lets us specify e.g. a car. But a configurator is not only helpful for these products with relatively few variables. Their function is to reduce the elicitation of the customer. Communication between buyer and supplier has proven to be critical for the value of customisation [Gilmore \& Pine 1997]. Configuration can be difficult and customers often have difficulties formulating needs and making decisions regarding a product [Zipkin 2001]. The consequences of a decision are often unclear, and the number of options can be overwhelming. The configurator can help guide the customer through the process and eliminate uncertainty and create an overview of options. Because, in spite of all the excitement over new variation options and features, it is clear that "customers do not want choice; they want what they want" [Pine 1994]. For efficient customization it is important to use the resources of $R \& D$ efficiently and therefore the number of trivial specification task must be limited to a minimum. 
For some manufacturers of customised goods the workload of producing tenders or bids is becoming a burden. As competition increases, the bid to order ratio falls and as a result bid costs are distributed fewer orders. As a result manufacturers have an interest in producing bids precisely and efficient. This can be done by making the knowledge of product development directly accessible to the salesperson, thereby reducing the cost of transferring information, and freeing resources from time consuming inquiries.

With complex products this is a significant problem since much of the engineering task often is placed in the proposal phase. To reduce costs some manufacturers choose to use configuration technology and thereby make it is possible with relative little effort to make a proposal reusing experience form earlier projects. At present much valuable R\&D time is spent on validating orders and compensating for inadequate documentation etc. And this is hollowing out the competitiveness of build to order production.

By focusing on keeping the every day operations out of $R \& D$ it is possible to focus on new and innovative designs that will create a lasting competitive advantage for the product program as a whole, rather than optimising on the individual order. As illustrated in Figure 2 the goal is to let the configurator hold the knowledge regarding the product.

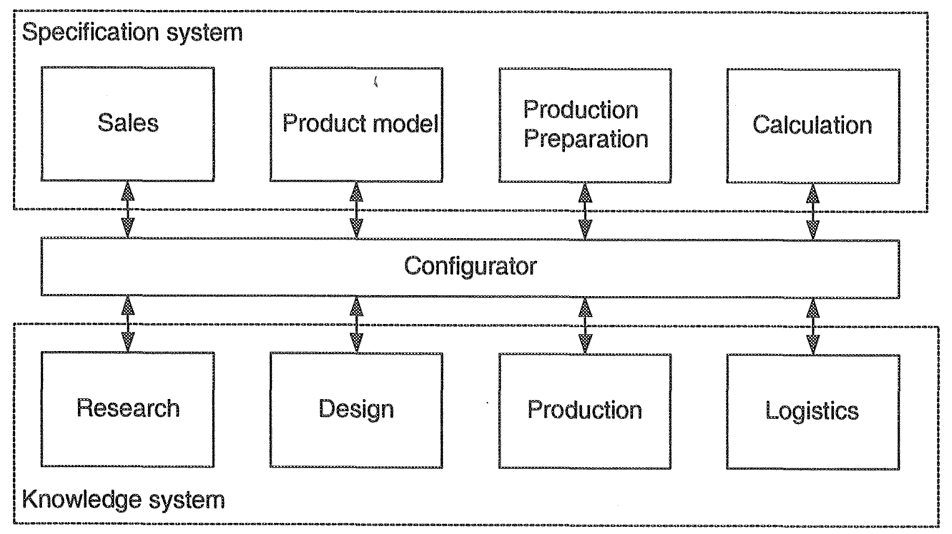

Figure 2 - The configurator

The specification system handles the processing of the individual order, where as the operational system the product-program as a whole is being improved. By applying a configurator the knowledge system is freed from the trivial order processing task.

If the implementation of a configurator shall be possible the products must have a significant similarity, otherwise it impossible to create a cost-efficient solution. Examples of industries where configurators have been applied are: Ships, manufacturing equipment and whole factories. In these cases internal configurators have proven effective and have reduced uncertainty and improved the transparency of operations by easy simulation consequences of changes. FLS industries' is an example of this complex configuration as described by [Hvam et al. 1999]. FLS designs and builds cement plants, which costs around 100 million USD. The factories are highly customised to meet local conditions in raw material. Producing 
tenders is a major cost driver due to the complexity of the product, but the implementation of a configurator has enabled FLS to apply an IT-supported preparation of tenders. Today, $80 \%$ of all orders go though the configurator and the results are as follows:

* Reduction of transition time from 5-20 days to $1-2$ days for the first generation of tenders

- Improved quality of quotes

* Reduction of resource consumption with a factor 25 in the preparation of a tender

The configurator has also proven to be an efficient tool for communication between the manufacturer and the customer because the customer can test various configurations and consequence calculations can be made very cheap, and thereby the best solution can more easily be found.

Other examples with similar results have been documented by [Svensson 2002 , Hansen 1999, Christiansen 1996, Hvam 1994]. By applying configuration sales forces have been able to create products that would technically satisfy customers. But configurators as they are generally known, represent a one way transport of static product information. Despite this amazing progress created by the implementation of configurators there is still some room for further progress.

Validating the structure of the product is only part of the potential embedded in a configurator. The product that the customer buys also contains important attributes such as price and delivery time, and these attributes can be important information in the sales situation. In order to enable these calculations, access to the inventory level and the availability of resources is needed. In the present situation these calculations are based on an average and experience, resulting in low profitability and late deliveries.

In relation to production, configuration of product only considers valid combination of items and components and does consider the planning and scheduling of the product! Rules regarding the psychical limitations of the production system are presented indirectly, in the limitations of the product. As a result the sales organisation often operates as an autonomous unit. An autonomous unit will naturally optimise to improve local efficiency. If the optimisation is to have the desired effect it must reflect reality. For a system as a whole local optimisation is not desirable, since it will reduce the overall efficiency, if the effect on the surrounding systems is not part of the decision foundation. Configuration and APS should therefore be linked together so that an ATP and CTP (available to promise and capable to promise) is available for order management in the sales situation, so that delivery times can be calculated and to this can a flexible cost structure be added.

\section{Configuration based on flexible cost structure}

Customised products mean that some of a production have to be produced, a therefore work in process is unavoidable, but depending on the degree of postponement the production time (lead-time) can vary. For some products the leadtime can be critical whereas it is less significant in other cases. But in both cases it is 
important to be able to give a precise estimation based on reality rather than intuition. This can save both the customer and manufacturer a lot of frustration and money.

Prices can be dependent on delivery time, especially if the product or component is built to order. Suppliers are often interested in getting a long leadtime, so that they have an opportunity to even out fluctuations in workload. If orders are given with short notice it is not possible for the supplier to optimise production over time and as a result costs will increase. To ensure overall efficiency, various forms of formalised collaboration are often established. In a typical buyer/supplier relationship the customer is obligated to buy a certain amount of components over the year. In return for the decreased flexibility the customer is rewarded with a low price. The supplier can use the order from the partner to fill out holes in the production plan, since it is certain to be sold within a year, and thereby the overall efficiency is increased, to the benefit of both parties.

\section{EVOLUTION OF INFORMATION SYSTEMS}

In this section an APS system is introduced and a short review of the development of information systems is presented. Secondly, a CTP in the APS system is presented together with a short comparison of ATP and CTP. Thirdly, in this section two models for CTP are discussed with some general considerations to make the right selection of CTP-model, and an example of the order promise of a customer order by use of product configuration and use of APS's CTP-model and functionality is given. Finally, a summary of the needed APS functionality in order management is given to improve order promise and to support the negotiation phase based on different delivery due dates.

Today's practice of supply chain planning was developed with an innovative manufacturing technique called material requirements planning. Although it is conceptually basic by today's standards, material requirements planning first introduced the benefits of planning to manufacturing managers all over the world and gave rise to a series of improvements in supply chain management, which are still valid today. Material requirement planning provides a time-phased view of component requirements over an extended horizon to give managers an early warning of potential inventory shortages at a manufacturing site [Vollmann et al. 1997]. Based on material requirements planning fundamentals, forecast scheduling, master production scheduling and capacity requirements planning were soon developed to supplement material requirements planning with high-level production planning, inventory management, and capacity analyses. All of these early planning tools generated unconstrained plans for a single manufacturing location. Manufacturing Resource Planning (MRPII) soon evolved to provide manufacturing planning and control mechanisms designed to monitor the progress of manufacturing execution and to compare with plans and schedules. MRPII was able to combine planning and execution and institute continuous feedback loops, but it was still limited to the activity within a single organization. In the early 1990 ERP emerged as an extension of MRPII, with the ability to integrate a company's manufacturing processes with its financial transactions. In the 1990s, advancements in technology 
and computing power spawned a new breed of planning systems: Advanced Planning and Scheduling (APS) systems [Steger-Jensen \& Hvolby 2001]. APS systems enable companies to reduce their cost of goods sold and to increase customer satisfaction by making more of the right products at the right times, using an optimal combination of manufacturing resources. Advanced planning systems utilize complex mathematical algorithms to forecast demand, to plan and to schedule production within specified constraints, and to derive optimal sourcing and productmix solutions. APS systems introduced the benefits of constraint based planning and optimisation to the business world, but the data intensive, cumbersome processes of typical APS applications limit the extensibility of solutions and usually restrict its use to a single organization or manufacturing site [Steger-Jensen and Hvolby, 2002 A], [Steger-Jensen and Hvolby, 2002 B].

Today, the Internet has changed the way business is conducted around the globe. The Internet enables companies to extend their market reach through new customer channels. Internet technologies provide an efficient, low cost medium for business communication and proliferation of data and, thus, enable multiple trading partners to collaborate on demand forecasts, production schedules and inventory requirements. Finally, companies which leverage Internet business practices throughout their business processes - such as new product development, supply chain management, and procurement - can realize extensive benefits, as for instance shortened time to market, increased visibility across the extended supply chain, and reduced costs.

\section{Order Promise with Capable-To-Promise functionality}

In this section an introduction to CTP in the APS system is presented. Then a short comparison of ATP and CTP is presented. In an APS system it is possible to evaluate and to simulate customer orders agents a plan and schedule, which gives order management increased visibility across the extended supply chain. Order promise is an important part of better integration between order management and production management. The initial approach to this order-promising problem was available-to-promise (ATP), which enabled the pledge of an order delivery based on future uncommitted inventory as projected by master production schedule (MPS). Although this approach has merit for a make-to-stock environment or if a forecasted customer demand has been included in the MPS, it is based on fixed lead times and infinite capacity assumptions of the MPS. In addition, it was unsuitable for a maketo-order environment. The obvious shortcoming that stems from ATP's infinite capacity assumption is addressed by CTP. The concept of projecting a quantity available for sale remains the same; however, by realistically considering manufacturing capacity constraints, order promising becomes more accurate.

Capable-to-promise (CTP) is a relatively new concept, developed in direct response to a rapidly changing manufacturing environment emphasising customer satisfaction to an unprecedented degree of customisation. It is often referred to as order promising, CTP is used for determination of when a new or unscheduled customer order can be delivered. CTP seems to have developed primarily in the context of advanced planning and scheduling (APS) systems, where it has been a common application related to order tracking [Hill 1999]. 
A short comparison of ATP and CTP shows that the calculated CTP sometime favourable (optimistic) as ATP promises, but they will be more accurate. On the other hand, CTP can be better than ATP, because it takes a broader view. CTP calculated across a multi-level supply chain can find some situations where customer demand can be fulfilled by utilising uncommitted capacity and materials from supply chain partners. CTP takes into consideration the predefined statement of current and future supply plus internal production capacity that is uncommitted plus Supplier material capacity that is uncommitted.

\section{CTP BASED ON AGGREGATE-CAPACITY MODEL VERSUS FINITE-CAPACITY SCHEDULING}

In this section a comparison of two different CTP models is presented and discussed, and some general considerations are made in order to make the right selection of the CTP-model. A CTP implementation employs a finite-capacity model of the manufacturing system to determine when an item can be delivered. By definition it includes any constraints that might restrict the production, such as availability of resources, lead-times for raw materials or purchased parts, and requirement for lower-level components or subassemblies. The resulting delivery date takes into account the production capacity, the current manufacturing environment, and future order commitments. The objective is to reduce the time spent by production planners in expediting orders and in adjusting plans due to inaccurate delivery date promises.

There is much discussion and disagreement on where the finite-capacity model used to determine promise dates must reside. ERP vendors maintain that an aggregate-capacity model (CM), which uses time buckets of capacity, provides sufficient detail to provide accurate dates. Finite-capacity scheduling (FCS) vendors claim that a model of the shop floor used for scheduling is required. The correct approach depends on the time frame of the decision, the complexity of the manufacturing system, and the level of detail of the capacity model. Up front, in order management, it must be clearly established to all users whether promise dates are operational (near-time) - directly affecting the current shop floor operation or whether they are more tactical (long-term) in nature.

The exact definitions of the operational and tactical time frames depend on the manufacturing environment, order promising practices, accuracy of forecasts, and manufacturing philosophy. Factors include manufacturing cycle times, raw material lead-times, manufacturing load, typical customer order lead-time, and the method used for setting order priorities at the shop floor. A simple way to define these time frames is to determine how far out in the future that a FCS system would have to look in order to schedule manufacturing orders accurately.

At present, a common definition of a CTP system does not exist. In reality, the requirement of most manufacturers would require a CTP system that is a hybrid of the FCS and CM approaches. The general flow starts with a customer asking the question, "Can you deliver an order by a specific date?" Or alternately, "When can you deliver this order?" The first step in answering either question is to release the order to the CTP system, which attempts to schedule the specified order (in a FCS 
model) or to check if the aggregate capacity is available (in a $\mathrm{CM}$ model). In the case of a FCS, the order can be scheduled either forward or backward. If the order has to be delivered by a specific date, the backward method is typically used for scheduling of the order as late as possible, while still fulfilling the customer's requested delivery date. If the order has to be delivered as soon as possible or cannot be scheduled using the backward method (for instance, due to lack of capacity), then the forward method must be used.

The aggregate CM approach is similar, is attempts to reserve the required capacity either as close as possible to the due date or as early as possible. Ideally, the system automatically considers both methods, depending on how the order is entered. If the customer requests a specific delivery date, the CTP analysis can either respond that the delivery date can be met, or that the order cannot be fulfilled as requested, but a later date can be met. For some organisations with simple manufacturing processes, this first level CTP capability provides a complete system. However, many manufacturing systems produce products requiring raw materials or purchased parts, and/or their products have a multilevel bill of material (BOM). This implies that the manufacturing capacity for these lower-level components or subassemblies must also be considered. Typically, these items must be modelled as hard constraints in the CTP model, generate and schedule their production or checking lead-times required to receiving the items (possibly spawning another set of CTP analyses with supplies). In a multilevel BOM, this analysis must be completed for all components. Other considerations, such as order-release quantities and lot sizing algorithms (order modifiers in general), must be incorporated in the CTP analysis, if appropriate. Finally, if required items will not be available on time, the company must adjust the delivery date to account for delays.

The accuracy of this CTP analysis is better in a FCS-based implementation, because it accounts for the exact production requirement and incorporates manufacturing protocols, such as lot sizing, setup constraints, and equipment availability. The $\mathrm{CM}$ approach considers many of these factors but typically at a less detailed level. A CM system may for example include all the capacity requirement of the lower-level components in the end item. This provides an aggregate capacity demand on manufacturing, but does not accurately consider timing requirements and other details of moving products through a shop floor.

A key question in selecting a CTP system is which type of model is required to provide accurate delivery or promise dates. APS systems are capable of evaluating CTP and ATP both according to planed and scheduled items and resources, and the whole supply chain too. Therefore, the best way to provide these accurate delivery dates at Internet speed is through an APS system which ties into the front-end order entry system, e.g. configurator, and slot the order based on realistic manufacturing projections provided though the CTP analysis. As focus on customer satisfaction increases, manufacturers who cannot consistently meet the promised delivery dates will suffer, since order fulfilment will become a qualifying attribute rather than a competitive advantage. In the end, the most significant barrier to success is viewing CTP as a stand-alone extension to an APS or ERP implementation. Instead, management must obtain support and cooperation from others in the organisation and envision the CTP initiative as an enabling technology, which will provide customers with rapid, accurate promises and on-time order delivery. 


\section{Application and Configuration of APS in order management}

In this section an example which illustrates the integration of product configuration and CTP in Order Management for Order Promising will be discussed.

The first step of the process is the product configuration. When Order management receives an order the following information is needed to fulfil the demand. First, the product's bill of material (BOM) must be designed through the product configuration. The second step is to pass the BOM information to the APS system where the delivery date and place, product specification and quantity, etc. are entered. Based on this information the APS system calculates a CTP and a cost function for the configuration according to the delivery date. In this situation the manufacturer will have a much more precise cost calculation than compared with a system without a CTP function. The manufacturer will be able to work with a well defined minimum price in the negotiation phase and, therefore, make rational decisions in contrast to earlier when the consequences of decisions made in the sales phase were unclear. CTP will not only calculate the workload but also provide other data. A CTP source will give the following result for order promise:

- view available quantity the request date

* view earliest date when requested quantity becomes available

* view CTP quantity, cumulative CTP quantity

- view set of sources by item

- view set of items by customer

* view by product family

If the available quantity, at the requested date is less then the requested quantity the order manager will also get information on the earliest date when the, requested quantity becomes available. For example, if a customer orders ten products $\mathrm{X}$, and the request date is: 10. September 2002, the APS system would replay; a result, which said that five products are available at the requested date, and all ten products are earliest available 15. September 2002.

If it is important that the customer gets the ten products on the requested date (10 September 2002), it is actually possible to simulate this condition. For example, it is possible to setup a condition according to having long time before supplier has to be notified if supplier's capacity has to be extended without cost increase, and this information is used in plan generation. For example if supplier requirement extends the available capacity at the supplier, a workflow can be setup, which sends a message to the supplier and ask for extension of the supplier's capacity before further planning or re-plan if the supplier is willing to extend the capacity. It is also possible to setup different transportation modes for items or services from subsuppliers and to rank both sub-suppliers and transportation modes, which are evaluated during plan generation.

Plan generation with optimisation turn-on is based on optimisation of the profit and reduction of the overall cost. APS can actually find out, which method is the cheapest way to deliver the costumer order by evaluating first the cost of extending the resource capacity, and second by evaluation the transport mode (e.g. by plane instead of by truck) from the supplier [Steger-Jensen and Hvolby, 2001]. 


\section{Problem with current CTP and order promise in APS}

APS does not give information about the production cost for the item, only the list price for the item and the total sum, which is unit list price multiplied with the item quantity. From a business point of view, the product revenue is based on production cost plus a profit margin $\%$. The problem is that the order promise function needs information about the production cost for the items too because depending on capacity constraint or sourcing from suppliers and/or transportation mode from supplier the cost can vary much compared to the list price of an item. Therefore, the consequence of not having production cost included in order promising, means that the profit margin \% could be very low or even negative. If a customer orders a product, which is not critical in time, the customer maybe prepared to order the product later in time, with reduced product price as payment. Therefore it is only a question of calculating and presenting the production cost, and product cost plus profit margin \%, at the order promise form, together with the different delivery alternatives.

\section{CONCLUSIONS}

This article is concluded as it started, namely with the statement that it is believe that the companies with the best ERP systems are best positioned to benefit from the integration of APS and configuration, because they can add the APS module on top of their ERP layer (or engine). The key to success is to ensure the functionality of both, because without a well working ERP system the APS will be more or less worthless. The traditional craft based customizers getting an ERP system can be a major challenge, since the procedures, structure and precision needed are so far from their technical level. For the manufacturers observed, the key to efficiency is to move focus from the completion of the individual order to a view of the overall production as a whole. When this is done, the tools suggested can help manufacturers of customised goods to reach new levels of efficiency. The benefit from using an integrated configuration/APS-system as described will be an opportunity to harvest the fruits of industrial production since many of the surprises linked to craft production will be eliminated. When these tools are combined, it will be possible to reach a situation where a precise calculation regarding the production cost can be simulated at an early stage and, thereby, it will be possible to make rational decisions in the negotiation phase and efficiently prioritise orders.

If order promise with ATP are based on infinite capacity or aggregated capacity, the inventory level and work in process (WIP) will increase, because the scheduling problem is not considered at all. This is necessary in order manufacturing environment, in general, because finale product inventory can not be used as buffer.

A make-to-order environment is generally highly dependent on data for order promise because it will no be possible to calculate the CTP without it. When order promise is based on more accurate data, the quality of the order promise will be more reliable and more effective. Not only will the customer services increase by better order promise tools such as the configurator and the APS, but also the cost related to order promise would be reduced significantly. 
A natural extension of the suggested system would be a close integration with suppliers. The perspectives are an integration of APS systems where it will be possible for the manufacturer to get ATP/CTP directly in the configuration situation, maybe even with multiple suppliers such that the supplier selection can also be integrated.

\section{REFERENCES}

[1] Christiansen, Kåre Groes; Concurrent Engineering - A knowledge Production Concept for a Shipyard; Odense Steel Shipyard, and Department of Industrial Management and Engineering, Technical University of Denmark, 1996.

[2] Davenport, Thomas; Enterprise systems and process change: sitll no quick fix; The financial times, 22 Feb., 1999, pp. $6-12$.

[3] Gilmore James $\mathrm{H}$ and Pine, Joseph, P.; The four faces of mass customisation; Harvard business review Jan - Feb 1997 vol. 75 no.1 pp. 91 - 102.

[4] Hansen, Benjamin and Hvam, Lars; Strategic guidelines for application of product models; The 4th annual international conference on industrial engineering theory applications and practice; San Antonio, Texas, 1999

[5] Hart, Christopher W.L.; Mass customisation: conceptual underpinnings opportunities and limits; International journal of service industry management, vol. 6, no. 21995 pp. $36-45$.

[6] Hill, Jr., Sidney, "A Bone Fide Fit?" Manufacturing Systems, April 1999, pp. 61 - 70.

[7] Hvam, Lars; Anvendelse af produktmodellering-set ud fra en arbejdsforberedelsessynsvinkel; Driftsteknisk Institut, DTU, 1994

[8] Hvam, Lars, Malis, Martin,Hansen Benjamin and Riis, Jesper; Reengineering of the quotation process - Application of knowledge based systems; 1999

[9] Hvam Lars, Riis Jesper, Malis Martin and Hansen Benjamin; A procedure for building product models; Proceedings, PM (Product models) Linköbing 2000

[10] Hvam, Lars and Have, Ulrik; Re-engineering the specification process; Business Process Management Journal, Vol. 4, No. 1, 1998

[11] Lampel, Joseph and Mintzberg, Henry; Customizing customisation; Sloan management review, fall 1996 pp. $21-30$.

[12] Pine, Joseph: Customers don't want choice; managers journal, Wall Street Journal 18. Apr, 1994, p A18.

[13] Sabin, Daniel and Weigel, Rainer; Product configuration frameworks - a survey; IEEE intelligent system July/August 199842 - 48

[14] Steger-Jensen, Kenn and Hvolby, Hans-Henrik; Analysis of the planning and scheduling functionality in APS systems, Proceedings of SPIE Internet-based Enterprise integration and management, Vol. 4566, 2001, pp. 109 - 16. ISBN: 0-8194-4294-1.

[15] Steger-Jensen, Kenn and Hvolby, Hans-Henrik; Constraint Based Planning in Commercial, Advanced Planning and Scheduling Systems, Proceedings of ICE Rome Italy, 2002

[16] Steger-Jensen, Kenn and Hvolby, Hans-Henrik, "Planning and Scheduling Strategies in SMEs" The Fifth SMESME International Conference, Clemsford United Kingdom, 2002

[17] Svensson, Carsten; The configurator that was never meant to be-Experiences from designing, implementing and using a low cost configurator, The Fifth SMESME International Conference, Clemsford United Kingdom, 2002

[18] Vollmann, T.E., Berry, W.L. and Whybark, D.C., Manufacturing Planning and Control Systems, Irwin, Homewood, IL, 1997. 
[19] Zipkin, Paul; The limits of mass customisation; Sloan management review, spring 2001, pp. $81-87$. 